\title{
FATORES QUE FAVORECEM E DIFICULTAM O TRABALHO DOS ENFERMEIROS NOS SERVIÇOS DE ATENÇÃO À SAÚDE
}

\author{
Factors that favor and hinder the work of nurses in services of health \\ attention \\ Factores que favorecen y dificultan el trabajo de los enfermeros en los \\ servicios de atención a la salud
}

\author{
Carmem Lúcia Colomé Beck \\ Francine Cassol Prestes ${ }^{4}$
}

Andrea Prochnow ${ }^{2}$

Rosângela Marion da Silva ${ }^{3}$

Juliana Petri Tavares ${ }^{5}$

\section{RESUMO}

0 enfermeiro depara-se com fatores que favorecem e dificultam a realização do seu trabalho e que interferem nos sentimentos em relação ao trabalho. Este estudo é do tipo exploratório-descritivo, com abordagem qualitativa. Teve como objetivos caracterizar sociodemograficamente os enfermeiros dos Serviços de Atenção à Saúde de um município do Estado do Rio Grande do Sul e identificar os fatores que favorecem e dificultam a realização do trabalho. Os dados foram coletados de março a julho de 2007 por meio de um questionário. A amostra foi composta por 24 enfermeiros. A análise dos dados possibilitou construir dois eixos temáticos: fatores que favorecem e que dificultam a realização do trabalho do enfermeiro. Os resultados sugerem que os enfermeiros percebem satisfação no trabalho. No entanto, é necessário repensar o processo de trabalho desse profissional para fortalecer as ações perante o usuário do Sistema Único de Saúde e buscar resolutividade diante das necessidades da comunidade.

Palavras-chave: Atenção Primária à Saúde. Serviços de Saúde. Saúde do Trabalhador

\begin{abstract}
The nurse faces factors that favor and hinder the performance of his work and interfere with the feelings before work. This is an exploratory-descriptive study with a qualitative approach. It aimed to sociodemographically characterize the nurses of the Services of Health Attention of a town in the State of Rio Grande do Sul and to identify the factors that favor and hinder the performance of the work. The data were collected from March to July 2007 through a survey. The sample was composed by 24 nurses. The data analysis enabled two build two theme axes: factors that favor and hinder the performance of nurse work. The results suggest that nurses perceive satisfaction at work. However, it is necessary to rethink the process of work of this Professional to strength the actions before the user of Single Health System's and search for resolutivity before the necessities of the community.
\end{abstract}

Keywords: Primary Health Care. Health Services. Occupational

\begin{abstract}
Resumen
El enfermero se depara con factores que favorecen y dificultan la realización de sus actividades laborales y que interfieren en los sentimientos relacionados al trabajo. Este estudio es de tipo exploratorio-descriptivo, con un abordaje cualitativo. Sus objetivos fueron caracterizar socio-demográficamente los enfermeros de los Servicios de Atención a la Salud de una ciudad de la provincia de Rio Grande do Sul e identificar los factores que favorecen y dificultan la realización del trabajo. La recolección de los datos se hizo de marzo hasta julio de 2007, por medio de un cuestionario. Compusieron la muestra 24 enfermeros. El análisis de los datos posibilitó construir ejes temáticos: factores que favorecen y que dificultan la realización del trabajo del enfermero. Los resultados indican que los enfermeros perciben la satisfacción en el trabajo. Sin embargo, es necesario repensar el proceso de trabajo de ese profesional para fortificar las acciones que desarrolla frente al usuario del Sistema Único de Salud y buscar soluciones delante de las necesidades de la comunidad.
\end{abstract}

Palabras clave: Atención Primaria de Salud. Servicios de Salud. Salud Laboral

\footnotetext{
${ }_{1}^{1}$ Enfermeira. Doutora em Enfermagem. Professor Associado I do Departamento de Enfermagem UFSM. Santa Maria-RS- Brasil. Membro do Grupo de Pesquisa "Trabalho, Saúde, Educação e Enfermagem, Coordenadora da linha de pesquisa "Saúde do Trabalhador". Santa Maria-RS-Brasil. E-mail: carmembeck@gmail.com. ${ }^{2}$ Acadêmica de Enfermagem. Bolsista de Iniciação Científica/ UFSM. Membro do Grupo de Pesquisa“Trabalho, Saúde, Educação e Enfermagem, linha de pesquisa "Saúde do Trabalhador".Santa Maria-RS-Brasil. E-mail:andrea-zinha@hotmail.com; ${ }^{3}$ Enfermeira. Mestre em Enfermagem. Membro do Grupo de Pesquisa "Trabalho, Saúde, Educação e Enfermagem, linha de pesquisa "Saúde do Trabalhador".Santa Maria-RS-Brasil. E-mail: rosangelamarion@smail.ufsm.br ${ }^{4}$ Enfermeira. Membro do Grupo de Pesquisa "Trabalho, Saúde, Educação e Enfermagem, linha de pesquisa "Saúde do Trabalhador".Santa Maria-RS- Brasil. Email: francassol@bol.com.br ${ }^{5}$ Enfermeira. Professora Substituta do Departamento de Enfermagem UFSM. Mestranda em Enfermagem pelo PPGEnf/UFSM. Membro do Grupo de Pesquisa "Trabalho, Saúde, Educação e Enfermagem, linha de pesquisa "Saúde do Trabalhador". Santa Maria-RS-Brasil. E-mail: jupetritavares@gmail.com
} 


\section{INTRODUÇÃO}

A partir da última década do século passado, o Brasil experimentou mudanças na organização, no financiamento e na oferta de serviços em seu sistema de saúde. Essas mudanças foram percebidas, em especial, na atenção básica, com a introdução de programas inovadores e estratégicos para a mudança no modelo assistencial do Sistema Único de Saúde (SUS). ${ }^{1}$

A atenção básica caracteriza-se como um conjunto de ações, de caráter individual ou coletivo, voltadas para a promoção da saúde, prevenção de agravos, tratamento e reabilitação. ${ }^{2}$ Seu conceito surgiu e se consolidou no contexto de ampliação da descentralização do SUS e na mudança do modelo assistencial, com o intuito de destacar as ações preventivas e de enfrentamento dos determinantes de saúde. ${ }^{3}$

0 enfermeiro que atua nos Serviços de Atenção à Saúde, tais como as Unidades Básicas de Saúde (UBS), as Unidades de Estratégia de Saúde da Família (ESF), os Serviços Especializados, os Complementares, os de Vigilância à Saúde e na Coordenadoria do Meio Ambiente e Saneamento, depara-se constantemente com fatores que favorecem ou dificultam a realização do seu trabalho. Dessa forma, esses fatores, em muitas situações, fazem emergir nesse trabalhador sentimentos ambíguos com relação ao trabalho, ou seja, podem fazê-lo compreender o trabalho como dual, com consequências de maior ou menor importância sobre a vida cotidiana. ${ }^{4}$

Estudo realizado em uma Unidade de Estratégia de Saúde da Família ${ }^{5}$ identificou que 0 enfermeiro tem, por um lado, facilidade em exercer a função de coordenação dentro das unidades de saúde por conhecer melhor o seu funcionamento global e por manter um bom relacionamento com toda a equipe; por outro, identificou como dificuldade a falta de capacitação para supervisionar e fazer grupos e a carência na formação acadêmica, além do acúmulo de funções, o que implica a falta de tempo para exercer suas atividades.

Com base no contexto apresentado, os objetivos deste estudo foram caracterizar sociodemograficamente os enfermeiros dos Serviços de Atenção à Saúde de um município localizado no interior do Estado do Rio Grande do Sul e identificar os fatores que favorecem e dificultam a realização do trabalho deste profissional. É relevante na medida em que os dados emergidos podem auxiliar no delineamento de estratégias para melhoria dos serviços de saúde de modo a possibilitar (re)pensar o processo de trabalho desses profissionais na busca do reconhecimento, da satisfação profissional e da qualidade da assistência prestada.

\section{PERCURSO METODOLÓGICO}

A pesquisa é do tipo exploratório-descritiva, com trajetória metodológica da pesquisa qualitativa. Foi realizada em um município com mais de 270 mil habitantes localizado no interior do Estado do Rio Grande do Sul.

Os sujeitos pesquisados foram os enfermeiros dos Serviços de Atenção à Saúde do município. Consideraram-se Serviços de Atenção à Saúde as Unidades Básicas de Saúde, as Unidades de Estratégias de Saúde da Família e os Serviços Especializados e Complementares, o Serviço de Vigilância à Saúde e a Coordenadoria do Meio Ambiente e Saneamento.

A coleta dos dados ocorreu no período de março a julho de 2007, sendo que, nesse período, dados da Secretaria de Saúde do município informavam que 52 enfermeiros trabalhavam nos Serviços de Atenção Básica.

Para a coleta dos dados, foi utilizado um questionário com questões fechadas, para a caracterização sociodemográfica e abertas, com questões específicas sobre os fatores que favorecem e que dificultam a realização do trabalho do enfermeiro.

Esse questionário foi entregue individualmente, durante o turno de trabalho, sendo agendada uma data para a devolução. Como critério de inclusão, adotou-se que seriam incluídos no estudo todos os enfermeiros que desejassem participar, que estavam em exercício no período de coleta de dados e que preenchessem integralmente 0 questionário.

Anterior à aplicação do questionário, foi realizado um estudo piloto com três enfermeiros para verificar se as questões do instrumento respondiam aos objetivos do estudo. Assim, por não haver necessidade de reformulação das questões, os questionários foram incluídos na análise dos dados.

Para a análise dos dados, utilizou-se a análise de conteúdo. ${ }^{7}$ Dentre as diversas modalidades desse tipo de análise, optou-se pela análise temática com base na regularidade das afirmações, denotando, dessa forma, estruturas de relevância, valores de referência e comportamentos presentes ou subjacentes nos discursos. ${ }^{8}$

Primeiramente, realizou-se a leitura dos dados na íntegra, para apreensão inicial de seus conteúdos, e estabeleceu-se que os dados seriam analisados em torno de dois eixos temáticos: fatores que favorecem e que dificultam a realização do trabalho do enfermeiro. A seguir, foram identificados e agrupados os dados que se repetiam e/ou possuíam semelhança semântica nos diferentes 
fragmentos de acordo com os objetivos do estudo. Posteriormente, realizou-se a categorização dos elementos constitutivos de cada tema de acordo com a repetição e homogeneidade dos dados.

Em consonância ao preconizado pela Resolução 196/ $96^{6}$, foram respeitados os preceitos da ética em pesquisa. Assim, foram explicados individualmente os objetivos do estudo e 0 caráter voluntário de participação a todos os enfermeiros. Aqueles que aceitaram participar da pesquisa, foram orientados a ler o Termo de Consentimento Livre e Esclarecido e, na concordância dos termos expostos, assiná-lo.

Essa pesquisa foi financiada com bolsa do Programa Institucional de Bolsas de Iniciação Científica (PIBIC) do Conselho Nacional de Desenvolvimento Científico e Tecnológico (CNPq) no ano de 2006 e foi submetida ao Comitê de Ética em Pesquisa da Universidade Federal de Santa Maria em 2007. Obteve parecer favorável do Certificado de Apresentação para Apreciação Ética (CAAE) sob n $0153.0 .243 .000-06$. Para preservar o anonimato dos enfermeiros, as informaç̃̃es obtidas foram codificadas utilizando-se números arábicos antecedidos pela letra "E" (E1, E2, E3...).

\section{APRESENTAÇÃO E DISCUSSÃO DOS RESULTADOS}

Foram distribuídos 52 questionários e desses 24 foram analisados, considerando-se os critérios de inclusão. A partir dos dados, identificou-se que a maioria dos enfermeiros é do sexo feminino $(95,8 \%)$ e que foram graduados entre os anos de 1995 e 2005 (41,7\%) conforme demonstra o Quadro 1. Esse dado revela que os enfermeiros tiveram sua formação norteada pelos princípios e diretrizes do Sistema Único de Saúde, o que pode ser um fator que favoreça o trabalho deles nas unidades abrangidas pelo sistema.

Quanto ao tempo de serviço na rede, $54,2 \%$ dos enfermeiros têm entre 1 e 9 anos. Esse dado possibilita afirmar que os enfermeiros, em sua maioria, podem perceber os impactos do sistema de forma diferente dos demais que estão há mais tempo nos serviços, pois ainda estão assimilando as normas e rotinas e, talvez, buscando novas formas de enfrentar as dificuldades.

Outro dado importante é que $75 \%$ dos enfermeiros informam participar de eventos científicos, o que demonstra que eles percebem a importância da atualização profissional, 0 que pode indiretamente apontar para a qualificação da assistência. A Secretaria de Saúde do município em estudo, além de considerar importante essa atualização profissional, apoia os trabalhadores quando possível, o que é entendido como um fator que pode contribuir para o sentimento de reconhecimento. Isso foi identificado em uma questão em que $58,3 \%$ dos enfermeiros informaram à secretaria o desejo de participar de eventos científicos e que $85,7 \%$ dos solicitantes obtiveram alguma forma de auxílio.
Quanto à capacitação, a maioria dos enfermeiros $(83,3 \%)$ possuía pós-graduação no momento de coleta dos dados. Desses, 68,2\% tinham especialização em Saúde Pública, Coletiva ou Saúde da Família; 22,7\% tinham outros tipos de especializações e 9,1\% tinham mestrado. Um percentual de $25 \%$ dos enfermeiros possuía mais de um curso de pósgraduação.

Os dados emergidos das questões abertas possibilitaram a construção de dois eixos temáticos: fatores que favorecem a realização do trabalho do enfermeiro e fatores que dificultam a sua realização.

Do eixo temático Fatores que favorecem a realização do trabalho do enfermeiro emergiram quatro categorias: Valorização e reconhecimento; Educação permanente; Vínculo estabelecido com a comunidade; e Trabalho conjunto com os Agentes Comunitários de Saúde (ACS).

Valorização e reconhecimento foi uma categoria apontada como sendo um fator que favorece o trabalho do enfermeiro. Houve referência de que o fato de o usuário procurar o serviço para obter orientações do enfermeiro é um ponto que repercute favoravelmente no sentimento de valorização e de reconhecimento do trabalho realizado. Isso pode ser percebido no fragmento a seguir:

\section{[...]0 ponto positivo que mais repercute em mim é a comprovação diária de que os usuários e a comunidade procuram na unidade, pela figura do enfermeiro em primeiro lugar, para resolver suas necessidades e obter orientações [...] (E1)}

A valorização do ser humano é uma fonte de motivação uma vez que é responsável por proporcionar espaço e incentivo ao trabalhador que compõe as organizações, o que pode implicar crescimento pessoal e profissional. ${ }^{9} \mathrm{~A}$ valorização e 0 reconhecimento profissional da enfermagem são desafios que devem ser enfrentados e que se encontram ancorados em resquícios históricos e em outros componentes sociopolíticos, os quais estruturam o papel social estabelecido para os enfermeiros na atualidade. ${ }^{10}$

Assim, embora o reconhecimento faça parte das expectativas de todos os que trabalham, esse raramente é conferido de modo satisfatório. Porém, quando a comunidade percebe o empenho contínuo dos profissionais por uma melhor qualidade no serviço e reconhece o seu papel frente à equipe, 0 trabalhador sente seu esforço valorizado. Isso se traduz afetivamente por um sentimento de alívio, de prazer, às vezes de leveza da alma ou de elevação. ${ }^{11}$

Educação permanente foi outra categoria encontrada como facilitadora no trabalho do enfermeiro. 0 aprimoramento é visto como enriquecedor da prática assistencial, o que pode contribuir para a qualidade no atendimento e ser uma facilidade no trabalho do enfermeiro, conforme o fragmento: 


\section{[...] ]é mais fácil trabalhar quando a equipe é capacitada para $o$ atendimento [...] (E13)}

A educação permanente parte do pressuposto da aprendizagem e propõe que a transformação das práticas profissionais esteja baseada na reflexão crítica sobre as práticas reais de profissionais reais em ação na rede de serviços. ${ }^{12}$ Essa educação deve começar desde o treinamento introdutório da equipe e operar por meio de todos os meios pedagógicos e de comunicação que forem possíveis, considerando-se as realidades de cada contexto, nisto incluindo-se a educação à distância. ${ }^{13}$

Além disso, os entrevistados apontaram que ter especialização em Saúde Pública ou Coletiva, somada a experiência adquirida na prática profissional, é um fator que facilita o desempenho das funções:

[...] a especialização que tenho na área de saúde pública, além do fato de eu possuir uma experiência de 15 anos em saúde pública, é uma facilidade que encontro em executar meu trabalho[...] (E3)

Deacordo com o Ministério da Saúde, a atualização técnicocientífica é apenas um dos aspectos da transformação das práticas, e não seu foco central, uma vez que a formação e o desenvolvimento englobam aspectos de produção de subjetividade, de habilidades técnicas e de conhecimento do SUS. ${ }^{12}$

Na categoria Vínculo estabelecido com a comunidade, o enfermeiro percebe que o usuário estabelece vínculo e sente confiança no serviço ofertado conforme fragmento a seguir:

\section{[...] os pacientes demonstram confiança em mim; querem} ser atendidos exclusivamente por mim [...] (E12)

0 estabelecimento de vínculo entre 0 usuário e 0 trabalhador, muitas vezes, é baseado em receptividade, afetividade, aceitação e confiança. 0 vínculo do binômio usuárioserviço de saúde é propiciado pelo bom atendimento e pelo bom desempenho profissional. ${ }^{14}$

0 vínculo otimiza o processo da assistência e proporciona aos trabalhadores o conhecimento de seus clientes e das necessidades deles, o que pode facilitar o seu acesso. ${ }^{14}$ Além disso, um dos elementos fundamentais de um efetivo acolhimento é a responsabilização do trabalhador com o estado de saúde do usuário, que sente despertado um sentimento de confiança em relação à pessoa que presta a assistência.

Isso se traduz na participação da comunidade em seguir as orientações da equipe de enfermagem referentes aos cuidados e tratamentos prescritos. Pode-se dizer que 0 acolhimento mostra a postura dos trabalhadores da unidade no que se refere ao atendimento das necessidades de saúde do usuário. ${ }^{15} 0$ vínculo é responsável por dar mais segurança aos usuários, além de permitir que os trabalhadores do serviço os conheçam, para serem capazes de eleger melhor as prioridades no que se refere à saúde destes. ${ }^{14}$

Na categoria Trabalho conjunto com os Agentes Comunitários de Saúde, os enfermeiros reconhecem como sendo muito útil o trabalho dos agentes nas ações que são desenvolvidas na comunidade:

\section{[...] trabalho em equipe, com os Agentes Comunitários} de Saúde, é muito útil. Eles levam os problemas e as necessidades de cada comunidade, facilitando as ações que serão desenvolvidas [...] (E23)

Os agentes são um elo entre a comunidade e a equipe da unidade de saúde porque são pessoas que conhecem a comunidade e residem na área onde exercem seu trabalho. Eles são capacitados para reunir informações pertinentes à saúde da comunidade e levá-las aos demais membros da equipe de saúde. ${ }^{16}$ Desse modo, o trabalho em equipe é fundamental para o impacto das aç̃̃es na comunidade.

Assim, quanto aos fatores que favorecem a realização do trabalho, os enfermeiros referiram sentimentos que podem repercutir favoravelmente em suas ações e que, somadas, objetivam qualidade da assistência prestada.

No eixo temático Fatores que dificultam a realização do trabalho do enfermeiro, emergiram três categorias: Falta de recursos humanos, de materiais e equipamentos; Falta de compreensão e paciência da comunidade e Dificuldades relacionadas à área física delimitada.

A falta de recursos humanos, de materiais e equipamentos foi apontada como um dos fatores que dificultam o trabalho do enfermeiro, pois repercute no processo de trabalho e na satisfação do usuário com o serviço oferecido. Os fragmentos demonstram preocupação dos trabalhadores com o assunto:

[...] a falta de determinados materiais, de equipamentos e de medicamentos, dificulta o nosso trabalho [...] (E6)

[...]posso dizer que a falta de pessoal é que gera certa dificuldade de dar maior atenção a todos os pacientes, gerando uma certa pressa em certos atendimentos, 0 que torna o nosso trabalho mais difícil[...] (E12)

A falta de recursos humanos pode sobrecarregar os trabalhadores no plano físico ou mental, traduzindo-se em sofrimento. 0 sofrimento ou desgaste psíquico precisa ser identificado no cotidiano laboral, uma vez que pode comprometer a saúde do trabalhador. ${ }^{17}$

Falta de compreensão e paciência da comunidade foi outra categoria apontada como dificultadora no trabalho do enfermeiro. 0 fato de o trabalhador não poder resolver todos os problemas identificados no serviço, ou que não estejam vinculados ao que lhes compete, pode repercutir em incompreensão por parte dos usuários: 
[...] a comunidade age com falta de compreensão. Ela quer sempre total resolutividade do profissional [...] (E15)

[...] os pacientes não compreendem a equipe quando não há medicação básica ou material de curativo que não é fornecido por estar em falta[...] (E12)

Os fragmentos expressam preocupação, pois os enfermeiros enfrentam situações em que a decisão não depende deles. Sabe-se que o enfermeiro tem uma função fundamental na equipe de saúde, pois realiza o levantamento de necessidades por meio da aparência externa ou da subjetividade do ser humano. ${ }^{18}$

No entanto, por assumir responsabilidades e papéis que não lhes competem, o trabalhador pode sentir-se sobrecarregado, 0 que o conduz a um progressivo sofrimento. ${ }^{17}$

$\mathrm{Na}$ categoria Dificuldades relacionadas à área física delimitada, houve referência à extensa área geográfica que dificulta tanto o acesso do usuário ao serviço quanto do trabalhador na realização das visitas domiciliares:

[...] a área geográfica grande dificulta o acesso da população à Estratégia de Saúde da Família e da equipe nas visitas domiciliares [...] (E6)

Pode-se dizer que a territorialização é um dos pressupostos básicos do trabalho na Unidade de Estratégia de Saúde da Família, que trabalha com a definição de um território de abrangência, que significa a área sob sua responsabilidade. ${ }^{13}$ Porém, as dificuldades apresentadas em relação à territorialização relacionam-se à extensão dessas áreas e às dificuldades de acesso a elas.

Quanto aos fatores que dificultam a realização o trabalho dos enfermeiros, percebeu-se que algumas situações não dependem da atuação deles para serem modificadas. Essas situações associadas a outras identificadas podem tanto comprometer o sentimento do trabalhador com relação ao seu trabalho quanto sobrecarregá-lo por não visualizar mudanças.

Portanto, a análise dos dados no que se refere aos fatores que favorecem e que dificultam o trabalho do enfermeiro que atua em Serviços de Atenção à Saúde permite refletir que, em alguns aspectos, os enfermeiros percebem satisfação em seu trabalho. No entanto, é necessário (re)pensar o processo de trabalho desse profissional no intuito de fortalecer as ações perante o usuário do SUS e buscar resolutividade diante das necessidades dos usuários.

\section{CONSIDERAÇÕES FINAIS}

Ao final do estudo, conclui-se que, no trabalho do enfermeiro nos Serviços de Atenção à Saúde, os fatores que facilitam e dificultam a realização do trabalho podem ser determinantes no que se refereà saúde do trabalhador e qualidade do serviço oferecido à comunidade.
As expectativas do trabalhador, que quando contempladas podem ser traduzidas em sentimentos de prazer e satisfação, somadas a transformação da prática por meio da educação permanente, a confianç̧a estabelecida com a comunidade e o impacto das ações conjuntas com os agentes comunitários são fatores que contribuem para a qualidade da assistência prestada e para 0 sentimento de realização pessoal e profissional. É importante que o enfermeiro busque 0 aperfeiçoamento e que tenha experiência, conhecimento e incentivo para desempenhar suas atividades.

No entanto, no cotidiano laboral, há entraves, e esses podem ser traduzidos como problemas de difícil solução, o que pode comprometer a saúde do trabalhador e o desenvolvimento das atividades. Executar o trabalho na tentativa de burlar essas dificuldades, trabalhar com falta de recursos materiais e humanos, além do descontentamento da comunidade, é uma tarefa árdua e pode contribuir para a sobrecarga física e mental do trabalhador.

Compreende-se como uma limitação desse estudo o fato de não serem identificadas estratégias coletivas e individuais dos enfermeiros contra as adversidades encontradas no processo de trabalho.

Outro aspecto a ser observado é que, apesar das limitações impostas, a comunidade reconhece o trabalho do enfermeiro, o que o faz sentir-se valorizado, e isso pode ser traduzido em um trabalho prazeroso.

Porém, apesar das dificuldades, é imprescindível que a organização do trabalho construa coletivamente medidas de promoção à valorização e o reconhecimento dos seus trabalhadores, o que poderá contribuir no sentimento de satisfação e alegria no desempenho das atividades.

Quadro 1 - Perfil dos enfermeiros de um Serviço de Atenção à Saúde, RS, 2007.

\begin{tabular}{|lll|}
\hline Variável & Frequência & Percentual \\
\hline Sexo & & \\
Feminino & 23 & $95,8 \%$ \\
Masculino & 1 & $4,2 \%$ \\
Ano de formação & & \\
& & \\
1975 e 1984 & 5 & $20,8 \%$ \\
1985 e 1994 & 9 & $37,5 \%$ \\
1995 e 2005 & 10 & $41,7 \%$ \\
Pós-Graduação & & \\
Sim & 20 & $83,3 \%$ \\
Não & 4 & $16,7 \%$ \\
Tempo de serviço na rede & & \\
1 - 9 anos & & \\
$10-19$ anos & 13 & \\
$30-39$ anos & 10 & $41,7 \%$ \\
Participação em eventos científicos & & \\
Sim & 1 & \\
Não & 18 & \\
\hline Total & 6 & \\
\hline
\end{tabular}




\section{REFERÊNCIAS}

1. Macinko J, Almeida C, Oliveira E. Avaliação das características organizacionais dos serviços de atenção básica em Petrópolis: teste de uma metodologia. Saúde em Debate [periódico na Internet]. 2003 set/ dez; [citado 22 set 2009]; 27(65): 243-56. Disponível em: http:// cebes.org.br/media/File/publicacoes/Rev\%20Saude\%20Debate/ Saude\%20em\%20Debate_n65.pdf.

2. Portaria $n^{0}$. 3.925, de 13 de novembro de 1998. Aprova o Manual para Organização da Atenção Básica no Sistema Único de Saúde. Diário Oficial da Republica Federativa do Brasil, Brasília (DF), 17 nov 1998. Disponível em:http://dtr2004.saude.gov.br/dab/docs/legislacao/ portaria3925_13_11_98.pdf.

3. David HMSL, Mauro MYC, Silva VG, Pinheiro MAS, Silva FH. Organização do trabalho de enfermagem na atenção básica: uma questão para a saúde do trabalhador. Texto\& Contexto Enferm 2009 abr/jun; 18(2): 206-14.

4. Beck CLC. 0 sofrimento do trabalhador: da banalização a resignificação ética na organização da enfermagem. Florianópolis (SC): UFSC; 2001.

5. Santos MR. Atribuições legais do enfermeiro no Programa Saúde da Família: dificuldades e facilidades. Bol Saude. 2003 jul/dez; 17(2): 3740.

6. Resolução ${ }^{0} 196$, de 10 de outubro de 1996 que Aprova as diretrizes e normas regulamentadoras de pesquisas envolvendo seres humanos. [on-line] [12 jul 2002]. Disponível: http://conselho.saude.gov.br/ resolucoes/ 1996/Reso196.doc

7. Bardin L. Análise de conteúdo. Lisboa(PO): Ed 70; 2007.

8. Minayo MCS. 0 desafio do conhecimento: pesquisa qualitativa em saúde. 10ªed. São Paulo (SP): Hucitec; 2007.

9. Beck CLC, Lisbôa RL, Tavares JP, et al. Humanização da assistência de enfermagem: percepção de enfermeiros nos serviços de saúde de um município. Rev Gaucha Enferm 2009 mar; 30(1): 54-61.

10. Beck CLC, Prestes FC, Tavares JP, et al. Identidade profissional dos enfermeiros de serviços de saúde municipal. Cogitare Enferm 2009 jan/ mar; 14(1): 114-9.

11. Dejours C. A banalização da injustiça social. Rio de Janeiro(RJ): Fundação Getúlio Vargas; 2007.

12. Ministério da Saúde (BR). Departamento de Gestão e da Educação na Saúde. Política de educação e desenvolvimento para o SUS: caminhos para a educação permanente em saúde. Pólos de Educação Permanente em Saúde. Brasília (DF); 2003.

13. Ministério da Saúde (BR). Saúde da família: uma estratégia para a reorientação do modelo assistencial. Brasília (DF); 1997.

14. Lima MADS, Ramos DD, Rosa RB, et al. Acesso e acolhimento em unidades de saúde na visão dos usuários. Acta Paul Enferm 2007; 20(1): 12-7.
15. Ramos DD, Lima MADS. Acesso e acolhimento aos usuários em uma unidade de saúde de Porto Alegre, Rio Grande do Sul, Brasil. Cad Saude Publica 2003 jan/fev; 19(1): 27-34.

16. Ministério da Saúde (BR).Guia Prático do Programa de Saúde da Família. Brasília (DF); 2001.

17. Silva RM, Beck CLC, Guido LA, et al. Facilidades e dificuldades enfrentadas por enfermeiros no trabalho noturno: estudo qualitativo. Online Brazilian J Nurs [periódico na internet]. 2009 jul; [citado 23 set 2009]. Disponível em: http://www.uff.br/objnursing/index.php/nursing/ article/view/j.1676-4285.2009.2346.

18. Balduino AFA, Mantovani MF, Lacerda MR. 0 processo de cuidar de enfermagem ao portador de doença crônica cardíaca. Esc Anna Nery Rev Enferm 2009; 13(2): 342-51. 